THE ARTS

CHILD POLICY

CIVIL JUSTICE

EDUCATION

ENERGY AND ENVIRONMENT

HEALTH AND HEALTH CARE

INTERNATIONAL AFFAIRS

NATIONAL SECURITY

POPULATION AND AGING

PUBLIC SAFETY

SCIENCE AND TECHNOLOGY

SUBSTANCE ABUSE

TERRORISM AND HOMELAND SECURITY

TRANSPORTATION AND INFRASTRUCTURE

WORKFORCE AND WORKPLACE
This PDF document was made available from www.rand.org as a public service of the RAND Corporation.

Jump down to document $\nabla$

The RAND Corporation is a nonprofit research organization providing objective analysis and effective solutions that address the challenges facing the public and private sectors around the world.

\section{Support RAND}

$\underline{\text { Browse Books \& Publications }}$

Make a charitable contribution

\section{For More Information}

Visit RAND at www.rand.org

Explore RAND Testimony

View document details
Limited Electronic Distribution Rights

This document and trademark(s) contained herein are protected by law as indicated in a notice appearing later in this work. This electronic representation of RAND intellectual property is provided for non-commercial use only. Unauthorized posting of RAND PDFs to a non-RAND Web site is prohibited. RAND PDFs are protected under copyright law. Permission is required from RAND to reproduce, or reuse in another form, any of our research documents for commercial use. For information on reprint and linking permissions, please see RAND Permissions. 


\title{
The Case for Keeping Quality on the Health Reform Agenda
}

\author{
ELIZABETH A. MCGLYNN
}

CT-306

June 2008

Testimony presented before the Senate Committee on Finance on June 3, 2008

This product is part of the RAND

Corporation testimony series.

RAND testimonies record testimony presented by RAND associates to federal, state, or local legislative committees; government-appointed commissions and panels; and private review and oversight bodies. The RAND Corporation is a nonprofit research organization providing objective analysis and effective solutions that address the challenges facing the public and private sectors around the world. RAND's publications do not necessarily reflect the opinions of its research clients and sponsors. RAND ${ }^{\circledR}$ is a registered trademark. 
Published 2008 by the RAND Corporation 1776 Main Street, P.O. Box 2138, Santa Monica, CA 90407-2138

1200 South Hayes Street, Arlington, VA 22202-5050 4570 Fifth Avenue, Suite 600, Pittsburgh, PA 15213-2665

RAND URL: http:/ / www.rand.org

To order RAND documents or to obtain additional information, contact

Distribution Services: Telephone: (310) 451-7002;

Fax: (310) 451-6915; Email: order@rand.org 


\title{
Elizabeth A. McGlynn, Ph.D. ${ }^{1}$ \\ The RAND Corporation
}

\section{The Case for Keeping Quality on the Health Reform Agenda ${ }^{2}$ \\ Before the Committee on Finance \\ United States Senate}

\begin{abstract}
June 3, 2008
Chairman Baucus, Ranking Member Grassley, and members of the Committee on Finance, I am honored to have the opportunity to testify before you today about the problems with health care quality. On a personal note, I am delighted to appear before my Colorado College classmate, Senator Salazar. Go Tigers!
\end{abstract}

I applaud the Committee for including quality as a topic for consideration in the debate about health care reform. All too often people assume that if we take care of the problems with cost and access, the quality problem will solve itself. The most important message I can leave you with today is this: if we find solutions to the problems of uninsurance and underinsurance and we control rising health care costs we will not have solved the quality problem. It is a separate problem. It requires separate solutions.

My testimony today covers three main areas. First, I will review with you some of the evidence about the problems with quality. I define quality as occurring when the right care is delivered to the right person at the right time, every time. Second, I will discuss some of the reasons these problems with quality exist. Third, I will highlight promising directions for solutions to these problems.

\section{The Quality Problem}

It is commonly said that the United States has the best health care system in the world. Although on many levels that is true, this assertion ignores the substantial gap that exists between what we know works and what is provided in the U.S. health care system. In 2003, my colleagues and I published in the New England Journal of Medicine, the first national, comprehensive study on quality of care for adults. We examined 439 indicators of quality for 30 clinical areas. We found that,

\footnotetext{
${ }^{1}$ The opinions and conclusions expressed in this testimony are the author's alone and should not be interpreted as representing those of RAND or any of the sponsors of its research. This product is part of the RAND Corporation testimony series. RAND testimonies record testimony presented by RAND associates to federal, state, or local legislative committees; government-appointed commissions and panels; and private review and oversight bodies. The RAND Corporation is a nonprofit research organization providing objective analysis and effective solutions that address the challenges facing the public and private sectors around the world. RAND's publications do not necessarily reflect the opinions of its research clients and sponsors.

${ }^{2}$ This testimony is available for free download at http://www.rand.org/pubs/testimonies/CT306/.
} 
on average, American adults received just $55 \%$ of recommended care for the leading causes of death and disability. To make that more concrete, participants in our study needed an average of 16 health care services - like mammograms to screen for breast cancer, flu shots, and medications to control blood pressure and blood sugar -- over a two year period and they received about 8 of those services. We spend nearly $\$ 2$ trillion annually on health care and we get it right about half the time. That may be the best in the world, but I think you would agree that we can and should do better.

We found problems across the board with preventive care (like flu shots), care for acute health problems (like colds), and care for chronic conditions (like diabetes). Adults received $55 \%$ of needed preventive care services, $54 \%$ of recommended care for acute health problems, and $56 \%$ of the care that doctors agree is necessary for people with chronic conditions.

Looking at the continuum of care, we found adults received $52 \%$ of needed screening services (like pap smears to screen for cervical cancer), $56 \%$ of services to accurately diagnose illnesses (like testing the lung function of people with asthma), $58 \%$ of needed treatments (like prescribing the right antibiotic for pneumonia), and $59 \%$ of needed follow-up care (like monitoring whether treatment for diabetes is effective in controlling blood sugar).

We evaluated how well the health care system delivered care for 30 common health care problems and found wide variation ranging from $79 \%$ of needed care for people with cataracts to $11 \%$ of needed care for persons identified by their doctors as having an alcohol dependence problem. In between those two extremes we found that $76 \%$ of needed care for breast cancer was delivered, but just $54 \%$ of needed care for asthma, $45 \%$ of needed care for diabetes, and $39 \%$ of needed care for pneumonia was delivered.

Colleagues of mine at RAND have published the findings of similar comprehensive studies of the quality of care delivered to vulnerable elders - people over the age of 75 who are at increased risk of significant declines in their health and functioning. Their results were similar to ours for problems common to all adults. But, for problems unique to the vulnerable elderly - like dementia, falls that can lead to hip fractures, and urinary incontinence - they found that these patients received just one-third of recommended care.

Last year, my colleagues and I published in the New England Journal of Medicine, results from a national study of the quality of care delivered to children. We found that, on average, children were receiving just $47 \%$ of recommended care overall. We were perhaps most surprised to find that children were receiving just $41 \%$ of the preventive services that they needed. Children with chronic illnesses received about $53 \%$ of needed services. The best performance was in care for acute 
health problems (colds, urinary tract infections) where we found that $68 \%$ of needed care was delivered.

At the time, people said to us - "well, there may be problems across the country, but the care in my local area is much better than that." We had collected enough information to allow us to construct quality scores for 12 metropolitan areas around the country. We found remarkably little variation in quality, ranging from $51 \%$ of recommended care delivered in Little Rock in Senator Lincoln's home state to $59 \%$ of recommended care delivered in Seattle in Senator Cantwell's home state. When we published these findings, the Seattle Post Intelligencer headline read "Seattle Best of A Bad Lot" - I thought they got it about right. I'm sure Senator Kerry would be disappointed to find that Boston, a well-known medical mecca, was second to Seattle at $57 \%$. Performance in other communities located in states represented on this committee included: $56 \%$ in Syracuse and $55 \%$ in Lansing and Phoenix.

The communities we studied were very different in terms of median income, the proportion of the population living below the poverty level, the proportion of the population lacking insurance, the number of hospital beds and physicians per 1000 population, and the penetration of managed care. These factors had no relationship to the quality of care delivered in the community. The relatively small differences we found in these very disparate communities have led most people who have looked at our study to conclude that their own community probably performs similarly.

The next comment we heard was - "well, quality may be a problem nationally and even in my community, but my care is excellent." In 2006, we published in the New England Journal of Medicine what we had found about who was at risk for receiving poor quality care. We found that everyone is at risk. We found no substantial advantages for population subgroups defined by gender, age, race, income, and insurance. We know these factors may make a difference in determining who gets in the door of the medical care system, but once in it appears that those factors convey little advantage.

The consequences of these failures to deliver needed care are significant. We found that $40 \%$ of persons with diabetes in our study had not had their blood sugar measured in the past two years. Such measurements are essential for tracking whether treatment regimens are effective - and for guiding changes to those regimens to prevent avoidable consequences. Among those who did have their blood sugar measured, nearly one-quarter had levels that were too high. Using models developed by the Diabetes Control and Complications Trial, we estimated that these quality gaps are associated with 2600 cases annually of preventable blindness and 29,000 cases annually of preventable kidney failure. 
We found that $58 \%$ of persons with hypertension in our study did not have their blood pressure adequately controlled. We estimated that this quality failure contributes to 68,000 preventable deaths annually from stroke, heart attacks, and other causes.

Among persons in our study who had a heart attack, 39\% failed to receive aspirin and $55 \%$ failed to receive beta blockers. These failures are associated with 37,000 preventable deaths annually many occurring in the 30 days following the heart attack. This is one area where we have seen substantial improvements thanks largely to routine monitoring and reporting of performance by the Centers for Medicare and Medicaid Services, the National Committee for Quality Assurance, and the Joint Commission.

Among those in our study over the age of 65 , more than one-third had not received a pneumococcal vaccine, which is effective in preventing the most common type of pneumonia. This level of performance is associated with 10,000 preventable deaths annually. On a side note, while we were doing this study much attention was given in the media to SARS - a disease we had no clear tools for prevention or treatment and which killed 813 people worldwide. Pneumonia - a disease we know how to prevent and treat and which affects many more people receives little public attention.

Finally, we found that $62 \%$ of study participants who were age 50 and older had not been screened for colon cancer using one of four methods that are known to be effective in early detection of this disease. This level of failure to screen for colon cancer and commence treatment is associated with 9600 preventable deaths annually.

Our colleagues studying the vulnerable elderly found that there was a significant association between the proportion of recommended care received and the likelihood that an individual would survive for the next three years. Poor quality causes premature death and disability.

In summary, there are significant gaps in the quality of care delivered nationally. Those gaps can be found in your community. Those gaps are likely to be experienced by you and the people most important to you. While we were conducting this study my father was hospitalized with congestive heart failure for the third time in about 18 months. In looking at his medical records (which he would only ask for after he was discharged from the hospital for fear of upsetting his doctor), we found that he was not on the right medications and he was on subtherapeutic doses of the medications he was receiving. My father was insured, educated and had been a hospital administrator for more than 30 years. And even he was failing to get the care he needed. 


\section{Why Is Quality So Poor?}

There is no single answer to the question - why is quality of care so poor? And it is tempting to point fingers in an effort to make the problem someone else's fault. But we are all part of the problem and we can all be part of the solution.

A starting point, and a key factor in motivating my colleagues and I to study this problem, is the lack of awareness about the quality gap among the public, health professionals, and policy makers. We are making strides in this regard but a lot of work remains to be done. The reason I believe we need to continue to raise public awareness is that if you do not believe you have a problem, you have no motivation to invest in finding and implementing solutions. For individuals, the lack of awareness can be dangerous - people may not take sufficient responsibility for making sure that they get the care they need. For health professionals, the lack of awareness may lead them to fail to demand access to the tools that can help close the gap. For policy makers, the lack of awareness can lead to failing to look for solutions and ignoring the policy options that could help stimulate improvement.

Another factor is the sheer explosion in knowledge about what is possible. In the last 5 years, for example, there were 4,424 articles registered on Medline reporting the results of clinical trials related to hypertension (and 22 review articles). For diabetes, 5,319 articles were published along with 10 review articles. For coronary artery bypass graft surgery, 1,453 articles were published and 5 review articles. Just reading the articles relevant to the diseases a physician is treating in his or her own practice would take more time than most practicing physicians have. But reading an article does not help one figure out (except rarely) whether one's practice should change as a result of the study. Review articles are designed to help provide summaries across large numbers of articles, but even those may not offer insights into how practice should change. Advances in science, while critically important and something we point to as an advantage of the American health care system, may be difficult to incorporate effectively into practice. One study found, for example, that it takes about 17 years on average for a new therapy to enter into mainstream practice. Our own study focused on the basics of good medical care. We did not look at how well medical care is delivered at the cutting edges, just at bread and butter medicine. And we found substantial failure on the basics. As knowledge accelerates, the gap is likely to widen.

Third, the U.S. health care system is a technological anomaly. We have made incredible advances in the availability of diagnostic machines, chemicals to treat or cure illnesses, microsurgical techniques to repair the ravages of disease or injury. Yet most doctors and hospitals rely on barely legible, handwritten notes to track what is done to a patient and how the patient responds to treatment. 
Doctors are also expected to maintain in their individual memories the appropriate approaches to diagnosing and treating a wide variety of diseases as they are manifest in human beings of radically different designs (age, race, height, weight, other health problems). By contrast, airline pilots are only allowed to fly one type of airplane and rely on extensive checklists and computer monitoring to ensure its safe operation. Nonetheless, we are surprised when physicians, using systems from the 19th century, and subject to the limitations of being human, fall substantially short of perfection.

Fourth, the health care delivery system itself is organized to deliver care reactively more than proactively. The delivery system reflects a time when the majority of health care problems were acute illnesses requiring the patient to show up at the doctor's office or hospital with symptoms. The health care system responded with what limited tools were at its disposal. As science has advanced and acute illnesses have given way to chronic diseases, the organization of care has not really changed. Patients initiate most of the health care visits with little prompting from their physicians. Patients often show up with multiple problems but no advance warning of what services they might require. The process is like running a meeting without an agenda (and often without notes about what happened at the last meeting). Visit lengths are short (17 minutes on average) and the patient and doctor may have very different objectives for the visit. When you add the individuality and complexity of most patients to the range of options for intervening, I think it is remarkable that the health care system gets it right as often as it does.

Finally, our methods of paying for health services are not aligned with the objectives of delivering high quality. We pay for piecework. We pay more money for more services whether or not they are needed. We may effectively pay less if a doctor keeps a patient healthy. We pay more for interventions than we do for thinking and talking to a patient. More recently there have been efforts to reward physicians who deliver better quality on select measures of performance. There is rarely enough money devoted to these strategies to offset the otherwise dysfunctional payment system. I do not think that the payment system caused the problem with quality, but I do believe that it presents a barrier to improving quality.

\section{So What Can Be Done?}

Although the focus of this hearing is on the problems of rising costs and low quality, I would like to offer some thoughts about solutions.

My colleagues and I conducted a study comparing quality of care in the Veterans Health Administration with that in the U.S. We were interested in studying the VHA experience because 
they have implemented many of the ideas that people have suggested might improve quality. They have one of the most mature electronic medical records systems in the country. The system includes reminders and protocols to help physicians deliver care consistent with standards. They routinely measure and report on the quality of care delivered in their facilities. Regional administrators have performance incentives as part of their compensation package.

We found that veterans receiving care in the VHA received recommended care about two-thirds of the time - significantly higher than the U.S. average. We found that the greatest difference was in those clinical areas where the VHA routinely measures and reports on quality, includes financial incentives for managers, and has decision support tools imbedded to facilitate clinical care. In those clinical areas where no measurement, incentives, or decision support was available, quality was still significantly better than the U.S. average, but the difference was relatively small. I think of this difference as the improvement you get in moving from paper records to electronic records.

I use this study to make a point: no single solution will close the quality gap. Rather we must engage in a sustained and multi-faceted approach to the problem. The VHA did not arrive at its superior performance overnight and we should not expect anything different from the rest of the health care system.

The starting point for improving quality is accelerating the adoption of interoperable, interconnected health information technology. We need this technology in every hospital, every doctor's office, every nursing home, every home health care provider, every school clinic. We need those systems to be able to talk to one another. We need health professionals to be trained to use the tools that can be imbedded in these technologies. And we need patients to have access to their own information. It is hard to imagine how any of the other solutions are possible or as effective in the absence of a modern information system. Such information systems will help physicians and patients more effectively partner to ensure optimal health. For this to happen, we need to invest not only in hardware and software, but also in "peopleware." If you look at the VHA and other systems that have successfully adopted information technology, they have also had large teams of people helping health professionals make the transition.

As a byproduct, widespread adoption of information systems will facilitate quality measurement, which in turn will enhance transparency efforts. Today, largely because of limits on the data systems in use, consumers have available relatively little information about the performance of different parts of the health care system. Reducing the burden of data collection (which will require enhanced and new functionality in health information systems) will increase the number of areas that can be measured and publicly reported on. It will mean that you and your family can get information that is relevant to your health concerns. 
We need to look at reforming the payment system to remove disincentives to improving quality. This likely will require fundamental overhaul, but there are clear examples of systems that have improved substantially even in the face of the current payment system. So, while it is not a rate limiting factor, it is likely that changes in payment policies will lead to accelerated quality improvement activities.

I would like to close with a final observation. The hearing today pairs the problem of rising health care costs with the problem of low quality. There is considerable support for the idea that improved quality will lower health care costs. While there are certainly examples of better quality being cheaper (where the quality problem is use of services that are not medically necessary), there are other areas where we would expect improved quality to increase costs (where the quality problem is the failure to deliver care that is known to be effective). The history of quality improvement for more than five decades shows greatest interest in improving quality to achieve cost reduction objectives. I hope this Committee has the courage to support efforts to improve quality whether or not it saves money. I hope you will be committed to improving quality because it is the right thing to do. It is possible that we as a nation will spend as much money as we are spending today - but we can substantially improve the value of that expenditure if we focus on making real the claim that America has the best health care system in the world. We can and we must deliver on that promise. Thank you. 